\title{
HIGH-MASS X-RAY BINARIES AND OB RUNAWAY STARS
}

\author{
L. Kaper, ${ }^{1}$ A. Van der Meer, ${ }^{1}$ and A. H. Tijani ${ }^{1}$
}

\section{RESUMEN}

Las binarias masivas de rayos X (HMXBs) representan una fase evolutiva importante, y proporcionan información sobre las propiedades de las primarias OB y de las secundarias compactas (estrellas de neutrones, agujeros negros). Observaciones recientes indican que las estrellas de neutrones en algunos sistemas (Vela X-1, $4 \mathrm{U}$ 1700-37) tienen masas mayores que la masa canónica $1.35 \mathrm{M}_{\odot}$. Estas observaciones tienen consecuencias importantes para la ecuación de estado a densidades supranucleares y para la formación de estrellas de neutrones y agujeros negros (supernovas y estallidos de rayos gama). A consecuencia de la explosión de supernova que produjo el objeto compacto, las HMXBs tienen velocidades espaciales altas, y son desbocadas. Alternativamente, las desbocadas $\mathrm{OB}$ pueden ser expelidas de un cúmulo mediante interacciones dinámicas. Las observaciones del Hipparcos indican que ambos mecanismos entran en juego.

\section{ABSTRACT}

High-mass X-ray binaries (HMXBs) represent an important phase in the evolution of massive binary systems and provide fundamental information on the properties of the OB-star primaries and their compact secondaries (neutron star, black hole). Recent observations indicate that the neutron stars in some of these systems (Vela X-1, 4U 1700-37) are more massive than the canonical mass of $1.35 \mathrm{M}_{\odot}$. These observations have important consequences for the equation of state at supranuclear densities and the formation mechanism(s) of neutron stars and black holes: supernovae and gamma-ray bursts. As a consequence of the supernova explosion that produced the compact star in these systems, HMXBs have high space velocities and thus are runaways. Alternatively, OB-runaway stars can be ejected from a cluster through dynamical interactions. Observations obtained with the Hipparcos satellite indicate that both scenarios are at work.

\section{Key Words: BINARIES: GENERAL - STARS: NEUTRON — STARS: SUPERGIANTS}

\section{HIGH-MASS X-RAY BINARIES}

In a high-mass X-ray binary (HMXB) a massive, OB-type star is orbited by a compact, accreting Xray source: a neutron star or a black hole. Mass is accreted either from the stellar wind of the massive companion (resulting in a modest X-ray luminosity of $\left.10^{35}-10^{36} \mathrm{erg} \mathrm{s}^{-1}\right)$, or flows with a higher rate to the compact star when the massive star fills its Roche lobe. In the latter case a much higher X-ray luminosity is achieved, $\sim 10^{38} \mathrm{erg} \mathrm{s}^{-1}$ close to its Eddington luminosity. Two classes of HMXBs can be distinguished based on the nature of the massive star: (i) the OB-supergiant systems, and (ii) the $\mathrm{Be} / \mathrm{X}$-ray binaries (for a catalogue of HMXBs, see Liu et al. 2000). The first class consists of the most massive systems, some of them containing a black hole (e.g. Cyg X-1). The second class comprises the majority $(\sim 80 \%)$ of the HMXBs which are often X-ray transients. The transient character is explained by the periodic increase in X-ray

\footnotetext{
${ }^{1}$ Astronomical Institute "Anton Pannekoek", University of Amsterdam, Kruislaan 403, 1098 SJ Amsterdam, The Netherlands (lexk@science.uva.nl).
}

luminosity when the neutron star, in its eccentric orbit, passes through periastron and accretes from the dense equatorial Be-star disk. Here, we concentrate on the OB-supergiant systems.

HMXBs are the descendants of massive binaries in which the initially most massive star (the primary) has become a neutron star or a black hole (Van den Heuvel \& Heise 1972). That the system remains bound after the supernova is due to a phase of mass transfer that occurs in the system when the primary grows larger than its Roche lobe. Note, however, that current binary evolution models including the effects of rotation and conservation of angular momentum indicate that in some systems the accretion efficiency must have been low (Langer et al. 2003). As a consequence of the mass transfer in the system, the secondary becomes the most massive of the two, so that with the supernova explosion of the primary less than half of the total system mass is lost and the system remains bound (Boersma 1961). It is assumed here that the supernova explosion is symmetric; in case of an asymmetry, the additional kick exerted on the compact remnant can cause the 
disruption of the system. Due to the loss of material (and momentum), the system gets a substantial "runaway" velocity (Blaauw 1961): on the order of $50 \mathrm{~km} \mathrm{~s}^{-1}$ for the most massive systems (and about $15 \mathrm{~km} \mathrm{~s}^{-1}$ for the less massive Be/X-ray binaries, cf. Van den Heuvel et al. 2001).

As long as the secondary is a massive main sequence star (and not a Be star), accretion of its relatively tenuous stellar wind onto the compact companion does not result in an observable X-ray flux. Only when the secondary becomes a supergiant, and subsequently when it overflows its Roche lobe, the accretion flow is dense enough to power a strong X-ray source; the system has become a HMXB. Most systems host an X-ray pulsar identifying the compact object as a neutron star. The HMXB phase lasts for a relatively short period of time ( 10,000 year): as soon as the Roche-lobe overflow commences, the orbit will shrink leading to an even higher mass transfer rate and a further tightening of the orbit. At some point, the X-ray source will be completely swamped with material optically thick in X-rays and/or penetrate the mantle of the secondary, followed by a rapid spiral-in. When also the secondary explodes as a supernova, a neutronstar binary (like the Hulse-Taylor binary pulsar) or two single neutron stars remain.

\section{PROPERTIES OF THE BINARY COMPONENTS}

Figure 1 gives an overview of the OB-supergiant systems in the Milky Way and the Magellanic Clouds (to scale). The orbital periods range from 1.4 (LMC X-4) to 41.5 days (GX301-2); the X-ray pulse periods from 0.71 (SMC X-1) to 860 seconds (2S 0114+065). A relatively long pulse period (minutes) indicates that the $\mathrm{X}$-ray source is a neutron star in a wind-fed system. The Roche-lobe overflow systems (e.g. Cen X-3) host rapid X-ray pulsars, most likely surrounded by an accretion disk. In these systems not only more mass, but also more angular momentum is transfered to the X-ray source, explaining the high (Eddington) $\mathrm{X}$-ray luminosity and rapid spin period.

\subsection{OB-supergiant primaries}

The mass of the $\mathrm{OB}$ supergiant primary and the compact companion in a HMXB can be accurately measured when the system hosts an X-ray pulsar. With the delay in pulse-arrival time and the radialvelocity curve of the OB supergiant the orbits of both stars are determined, and thereby their mass if the orbital inclination is known. The latter is well constrained if the system is eclipsing. In those systems,

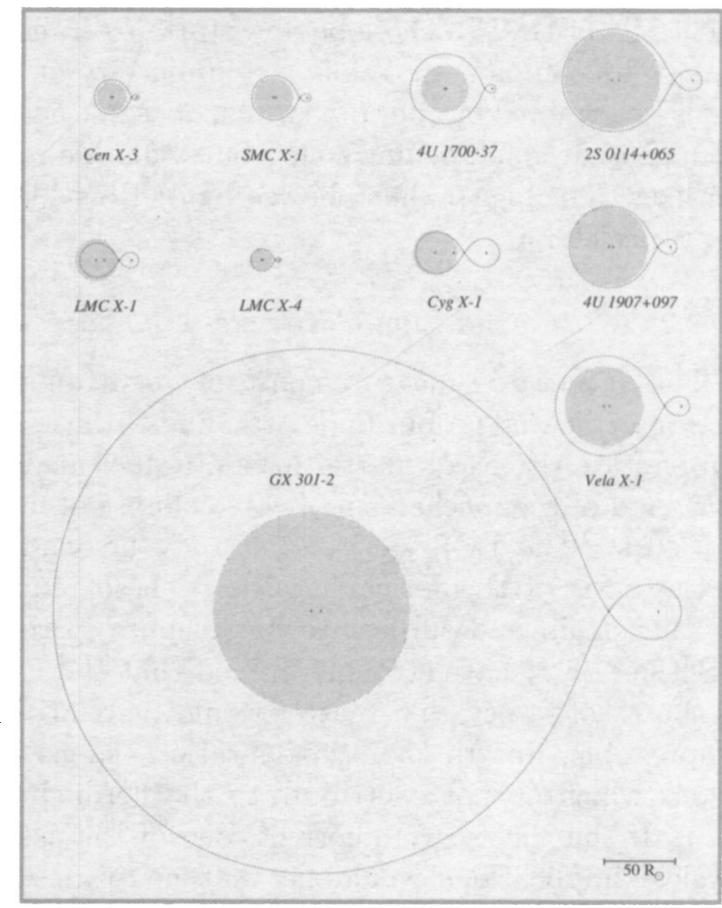

Fig. 1. An overview of OB-supergiant systems in the Milky Way and the Magellanic Clouds (not including $4 \mathrm{U}$ 1538-52 and LS 5039). The OB supergiants are (close to) filling their Roche lobe (drawn line). The Roche-lobe overflow systems (the X-ray pulsars Cen X-3, SMC X-1, LMC X-4, and very likely the black-hole systems LMC X1 and Cyg X-1) have circular orbits. The wind-fed systems (4U 1700-37, 2S 0114+065, 4U 1907+097, Vela X-1, and GX 301-2) have eccentric orbits, indicating that the orbits circularize with time and increasing mass-transfer rate.

also the radius of the OB supergiant can be derived with high precision from the duration of the X-ray eclipse. The mass ratio sets the size of the Roche lobe (e.g. Eggleton 1983); it turns out that the measured radii of the $O B$ supergiants are in very good agreement with the estimated size of the Roche lobe.

Earlier studies (e.g. Conti 1978, Rappaport \& Joss 1983) suggested that the OB supergiants in HMXBs are too luminous for their mass. E.g. the O6.5 giant companion of Cen X-3 has a mass of $19 \mathrm{M}_{\odot}$, while its luminosity corresponds to that of a star of more than $50 \mathrm{M}_{\odot}$. Besides this, the radius corresponding to the luminosity and effective temperature is larger than its measured (Roche-lobe) radius (cf. Kaper 2001). Thus, apart from being undermassive, the OB supergiants in HMXBs also seem to be undersized for their luminosity and temperature. This inconsistency is probably related to the phenomenon of Roche-lobe overflow. The OB star tries to become a supergiant, but at some point it 
reaches its critical Roche lobe and starts to transfer mass to its companion. While the luminosity of the star is determined by the core (which does not notice much of what is happening to the outer mantle), the star likes to be bigger than allowed by its Roche lobe and is peeled off.

\subsection{Neutron star and black hole secondaries}

The accurate measurement of neutron-star masses is essential for our understanding of the equation of state (EOS) of matter at supra-nuclear densities, and of the mechanism of core collapse of massive stars. The EOS can, so far, only be studied on the basis of theoretical models. These remain very uncertain and subject to hot dispute. Brown \& Bethe (1994) have strongly argued that the EOS must be "soft", i.e., that matter would be relatively compressible, due to kaon condensation (kaons are bosons which do not contribute to the Fermi pressure). If the theory were correct, one of the astrophysical implications would be that neutron stars cannot have a mass larger than $1.55 \mathrm{M}_{\odot}$; for larger masses, the object would collapse into a black hole.

The most accurate neutron-star masses have been derived for the binary radio pulsars. All of these are consistent with a small range near $1.35 \pm 0.04 \mathrm{M}_{\odot}$ (Thorsett \& Chakrabarty 1999). Recently, it has been shown that the neutron star in Vela X-1 is significantly more massive: $1.86 \pm 0.16 \mathrm{M}_{\odot}$ (Barziv et al. 2001, Quaintrell et al. 2003). Such a high neutron-star mass would rule out the soft equations of state. Also for $4 \mathrm{U}$ 1700-37 a high neutron-star mass is claimed (2.4 $\pm 0.3 \mathrm{M}_{\odot}$, Clark et al. 2002), although the X-ray source is, contrary to Vela X-1, not an X-ray pulsar (and perhaps a low-mass black hole). We (Van der Meer et al.) are currently analyzing the radial-velocity curves of other OB-supergiant systems with an (eclipsing) X-ray pulsar to find out whether Vela X-1 is an exception or that the neutron stars in these systems systematically deviate from the "canonical" mass of $1.35 \mathrm{M}_{\odot}$. This would provide an important constraint on the neutron-star formation mechanism (i.e. the supernova).

The estimated masses of black-hole candidates are substantially larger $\left(\sim 5-10 \mathrm{M}_{\odot}\right)$ than those measured for neutron stars. This suggests that neutron stars and black holes are formed in different ways. With the recent evidence that a black hole may be formed during the collapse of a massive star during a gamma-ray burst (GRB980425, Galama et al. 1998, Iwamoto et al. 1998), the hypothesis would be that neutron stars are formed in "ordinary" supernovae, while black holes originate from gamma-ray bursts.
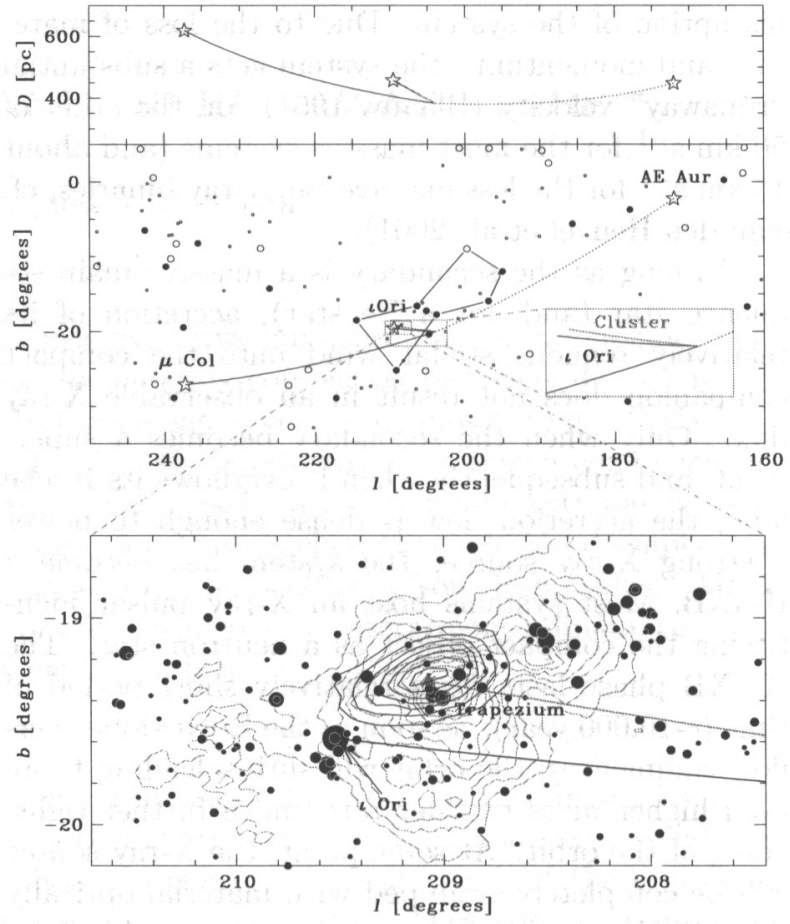

Fig. 2. Top \& middle: The orbits, calculated back in time, of the runaways AE Aur (dotted line) and $\mu \mathrm{Col}$ (solid line), and the binary $\iota$ Ori based on Hipparcos observations. The top panel shows the distance versus galactic longitude, the middle panel displays the orbits projected on the sky in galactic coordinates. The stars met $\sim 2.5$ Myr ago. Filled circles denote $O$ and B stars, open circles represent other spectral types. Bottom: A blow up of the central region shows the predicted position of the parent cluster (black contours) together with all stars in the Tycho Catalogue (ESA 1997) in the field down to $\mathrm{V}=12.4 \mathrm{mag}$. The brightest star is $\iota$ Ori; also the Trapezium is indicated. The grey contours (IRAS $100 \mu \mathrm{m} \mathrm{flux}$ ) outline the Orion Nebula. Figure from Hoogerwerf et al. (2000).

\section{OB-RUNAWAY STARS}

About $20 \%$ of the $\mathrm{O}$ stars, and a smaller fraction of the B stars, have a space velocity much higher than observed on average for the OB-star population in the Milky Way (about $10 \mathrm{~km} \mathrm{~s}^{-1}$, Stone 1979). Some OB stars have a space velocity exceeding $100 \mathrm{~km} \mathrm{~s}^{-1}$. Blaauw (1961) called these stars runaway stars, because at least for some of them the reconstructed path through space suggests an origin in a nearby $\mathrm{OB}$ association. How did these massive stars obtain such a high space velocity? Blaauw proposed that the supernova explosion of a massive companion in a binary results in a high velocity of the remaining massive star; the "modern" version of this Blaauw scenario is described in $\S 1$, which pre- 
dicts that all HMXBs are runaway systems. It took several decades to obtain the observational evidence proving that this scenario actually works (Van Rensbergen et al. 1996, Kaper et al. 1997); now, Hippar$\cos$ measurements have definitely confirmed the runaway nature of HMXBs (Chevalier \& Ilovaisky 1998, Kaper et al. 1999).

An alternative scenario for the formation of $\mathrm{OB}$ runaways is dynamical ejection from a compact cluster (Poveda et al. 1967, Portegies Zwart 2000). Due to the dynamical interaction between binaries (and single stars), now and then a massive star is ejected from an $\mathrm{OB}$ association. The probability for ejection is higher in dense starclusters; given the expansion of $\mathrm{OB}$ associations, one expects that dynamical ejection is most effective in young OB associations. An impressive example demonstrating the cluster ejection scenario is provided by the OB runaways AE Aur and $\mu$ Col. Blaauw \& Morgan (1954) noticed that these two stars move in almost opposite directions away from the Ori OB1 association and have the same kinematical age (i.e. the travel time from the association to its present location). Using Hipparcos observations, Hoogerwerf et al. (2000) managed to reconstruct the kinematical history of these two runaways in great detail and could show that both stars and the massive binary system $\iota$ Ori were at the same place in Ori OB1 $\sim 2.5$ million years ago (Fig. 3). The dynamical interaction between two binary systems apparently led to the disruption of one of them and the subsequent ejection of the two members (in opposite directions due to momentum conservation). Detailed dynamical simulations show that such an interaction is indeed possible (Gualandris et al. 2003). To match the estimated ages of the stars in the binary and its current eccentricity $(e=0.76)$, the authors suggest that the original members of the two interacting binaries were exchanged.

Hoogerwerf et al. (2000) studied the kinematical history of a sample of nearby runaways (17) and their (candidate) parent OB associations using Hipparcos data. A comparison of the kinematical age of the runaway and the age of the cluster is used to discriminate between the two formation scenarios. In case of dynamical ejection, the kinematical age of the runaway should be about equal to the age of the association. In the binary supernova scenario, the evolution of the binary system implies that the kinematical age of the runaway is significantly shorter than the age of the cluster. Furthermore, the $\mathrm{OB}$ runaway will be a blue straggler in the HRD of the cluster, because the OB runaway is rejuvenated (by gaining mass) during the first phase of mass transfer in the system. Hoogerwerf et al. conclude that the two mechanisms produce about equal amounts of runaways. This conclusion is in agreement with theoretical predictions (e.g. De Donder et al. 1997, Portegies Zwart 2000).

LK is supported by a KNAW fellowship and acknowledges financial support obtained from the LKBF.

\section{REFERENCES}

Barziv, O., et al. 2001, A\&A 377, 925

Blaauw, A. 1961, Bull. Astr. Inst. Neth. 15, 265

Blaauw, A., Morgan, W.W. 1954, ApJ 119, 625

Boersma., J. 1961, Bull. Astr. Inst. Neth. 15, 291

Brown, G.E, Bethe, H.A. 1994, ApJ 423, 659

Chevalier, C., Ilovaisky, S.A. 1998, A\&A 330, 201

Clark, J.S., et al. 2002, A\&A 392, 909

Conti, P.S. 1978, A\&A 63, 225

De Donder, E., Vanbeveren, D., Van Bever, J. 1997, A\&A 318,812

Eggleton, P. 1983, ApJ 268, 368

Galama, T.J., et al. 1998, Nat 395, 670

Gualandris, A., et al. 2003, MNRAS, submitted

Hoogerwerf, R., de Bruijne, J.H.J., de Zeeuw, P.T. 2000, ApJ 544, L133

Iwamoto, K., et al. 1998, Nat 395, 672

Kaper, L. 2001, in Proc. "Influence of binaries on stellar population studies", Ed. Vanbeveren, Kluwer Acad. Pub., p. 125

Kaper, L., Comerón, F., Barziv, O. 1999, in Proc. "WolfRayet Phenomena in Massive Stars and Starburst Galaxies", IAU Symp. 193, Eds. Van der Hucht, Koenigsberger, Eenens, p. 316

Kaper, L., et al. 1997, ApJ 475, L37

Langer, N., Wellstein, S., Petrovic, J. 2003, in Proc. IAU Symp. 212, Eds. K.A. van der Hucht, A. Herrero, C. Esteban, p. 275

Liu, Q.Z., Van Paradijs, J., Van den Heuvel, E.P.J. 2000, A\&AS 147, 25

Portegies Zwart, S.F. 2000, ApJ 544, 437

Poveda, A., Ruiz, J., Allen, C. 1967, Bol. Obs. Tonantzintla y Tacubaya 4,860

Quaintrell, H., et al. 2003, A\&A 401, 313

Rappaport, S.A., Joss, P.C. 1983, in Accretion-driven stellar $X$-ray sources, Eds. Lewin, Van den Heuvel, Cambridge Univ. Press, p. 1

Stone, R.C. 1979, ApJ 232, 520

Thorsett, S.E., Chakrabarty, D. 1999, ApJ 512, 288

Van den Heuvel, E.P.J., Heise, J. 1972, Nat 239, 67

Van den Heuvel, E.P.J., et al. 2001, A\&A 364, 563

Van Rensbergen, W., Vanbeveren, D., De Loore, C. 1996, A\&A 305,825 